\title{
Acute heart failure due to autoimmune myocarditis under pembrolizumab treatment for metastatic melanoma
}

\author{
Heinz Läubli ${ }^{1,4 \dagger}$, Cathrin Balmelli ${ }^{1 \dagger}$, Matthias Bossard ${ }^{2}$, Otmar Pfister ${ }^{2}$, Kathrin Glatz ${ }^{3}$ and Alfred Zippelius ${ }^{1,4^{*}}$
}

\begin{abstract}
Antibodies that stimulate the immune system by targeting inhibitory T cell receptors were successfully introduced into oncological practice and are capable to overcome tumor-induced immune evasion. In particular, targeting of the inhibitory receptors CTLA-4 and PD-1 or its ligand PD-L1 have been shown to be beneficial for patients with melanoma, renal cell cancer, non-small cell lung cancer and a growing list of other cancers with impressive response rates. Here, we report a severe, potentially life-threatening side effect of anti-PD-1 immunotherapy with pembrolizumab, which has not been previously described in the literature. A 73-year-old woman with metastatic uveal melanoma treated with pembrolizumab in third line developed severe heart failure due to pembrolizumab-mediated autoimmune myocarditis. Echocardiographic studies revealed a severely impaired left ventricular function with dyssynchrony. All tests for cardiotropic viruses were negative and histological analysis of a myocardial biopsy showed lymphocytic infiltration with a predominance of CD8 positive cells and a reduction of FOXP3 positive regulatory T cells. After initiation of corticosteroids and guideline-conform heart failure therapy, the symptoms rapidly improved and the left ventricular function recovered. While autoimmune myocarditis is a documented side effect of other checkpoint inhibitors, as for example ipilimumab and in one case with anti-PD-L1 antibody, it is not described for anti-PD-1-antibodies like pembrolizumab or nivolumab. As the FDA recently approved both pembrolizumab and nivolumab for melanoma progressing after anti-CTLA-4 treatment with ipilimumab, more patients will soon receive anti-PD-1 therapy. Thus, it is important to be aware of such rare, but severe immune-related adverse events.
\end{abstract}

Keywords: Melanoma, Immunotherapy, PD-1 blockade, Antibody, Nivolumab, Pembrolizumab, Autoimmunity, Anti-tumor T cell response, Myocarditis

\section{Background}

The field of cancer immunotherapy is currently moving forward at an accelerated pace. Though early clinical trials have yielded mixed results with ambiguous clinical benefit [1], cancer immunotherapy is now attracting increasing attention as viable therapeutic option, both in melanoma and in other malignancies [2]. In particular, recent therapeutic efforts targeting inhibitory receptors on $\mathrm{T}$ cells to overcome tumor-induced immune dysfunction has been successfully introduced into oncological practice. The clinical development of immune checkpoint blocking

\footnotetext{
* Correspondence: alfred.zippelius@usb.ch

${ }^{\dagger}$ Equal contributors

'Division of Medical Oncology, University Hospital Basel, Basel, Switzerland ${ }^{4}$ Department of Biomedicine, Cancer Immunology Laboratory, University of Basel, Basel, Switzerland

Full list of author information is available at the end of the article
}

antibodies has been pioneered by the antibody ipilimumab $\left(\right.$ Yervoy $\left.^{\circ}\right)$, which inhibits CTLA-4 and has demonstrated survival benefit in two randomized landmark trials in melanoma [3,4]. Capitalizing on this success, research on clinically relevant $\mathrm{T}$ cell checkpoint inhibition has been boosted. Early clinical trials have demonstrated meaningful response rates, sustained clinical benefits with exceptional survival rates and good tolerability of next-generation checkpoint inhibitors, including PD-1 and PD-L1 inhibitors across multiple cancer types [5-10]. Exciting perspectives include the concurrent blockade of different immunologic (non-redundant) checkpoints. The feasibility of this approach has recently been demonstrated in melanoma using combined CTLA-4 and PD-1 inhibition [11].

Inhibition of immune checkpoints induces side effects defined as "immune-related adverse events" (irAEs) [12]. 
Autoimmunity is the suggested mechanism sustaining these toxicities. Such irAEs often affect the skin, the intestinal mucosa or endocrine organs. Here, we report a case of autoimmune myocarditis with consecutive heart failure after treatment targeting PD-1.

\section{Case presentation}

A 73-year-old woman with metastatic uveal melanoma presented for a clinical examination and laboratory control under third-line therapy with pembrolizumab (MK-3475) in our oncological outpatient department. She suffered from progressive, severe dyspnea. Fourteen years earlier, a malignant uveal melanoma of her left eye was diagnosed and treated by ablative proton therapy. In February 2013, a relapse with hepatic metastases was diagnosed. No BRAF or NRAS mutations were found in the liver biopsy in accordance with low frequency of such mutations in uveal melanoma. The patient had no prior history of autoimmune or allergic disease. The hepatic lesions showed progressive disease after treatment with two cycles of dacarbazine, and, subsequently, four cycles with the anti-CTLA-4 antibody ipilimumab. Importantly, the latter therapy was tolerated without any adverse events. To improve local control, selective internal radiation therapy (SIRT) of the largest liver lesions was performed in December 2013. However, computed tomography 6 months later showed new bone, pulmonary, mesenterial and peritoneal metastasis (Figure 1). Palliative radiation therapy of symptomatic, lytic bone lesions was performed and denosumab monthly was started. Moreover, anti-PD1 therapy with pembrolizumab was initiated one month later at a dose of $2 \mathrm{mg}$ per kilogram body weight every third week, which led to stabilization of the disease after 8 weeks. The patient had no history of cardiac disease.

When she presented with progressive dyspnea (New York Heart Association Stage (NYHA) IV), she had received five cycles of pembrolizumab. Her prior medical history was free of any heart diseases or corresponding symptoms. Clinical examination now revealed congested neck veins, bilateral rales and lower leg edema. The electrocardiogram (ECG) showed a tachycardiac sinus rhythm with ventricular bigemy (Figure 2). Brain natriuretic peptide (BNP) and high-sensitivity troponin $\mathrm{T}$ (hs-TnT) levels were elevated (928 ng/L and $0.63 \mu \mathrm{g} / \mathrm{L}$, respectively). Thyroid stimulating hormone levels were within normal range $(2.65 \mathrm{mlU} / \mathrm{l})$. A pulmonary embolism or pneumonitis was excluded by computed tomography. The transthoracic echocardiography revealed a severely impaired left ventricular ejection fraction (LVEF) of 30\% with marked ventricular dyssynchrony (Movie Clip, Additional files 1-3). Taken together, the clinical presentation corresponded to acute heart failure.

The cardiac MRI did not show any signs consistent with an acute myocarditis or myocardial ischemia. Confronted with an unclear acute heart failure under immunotherapy,

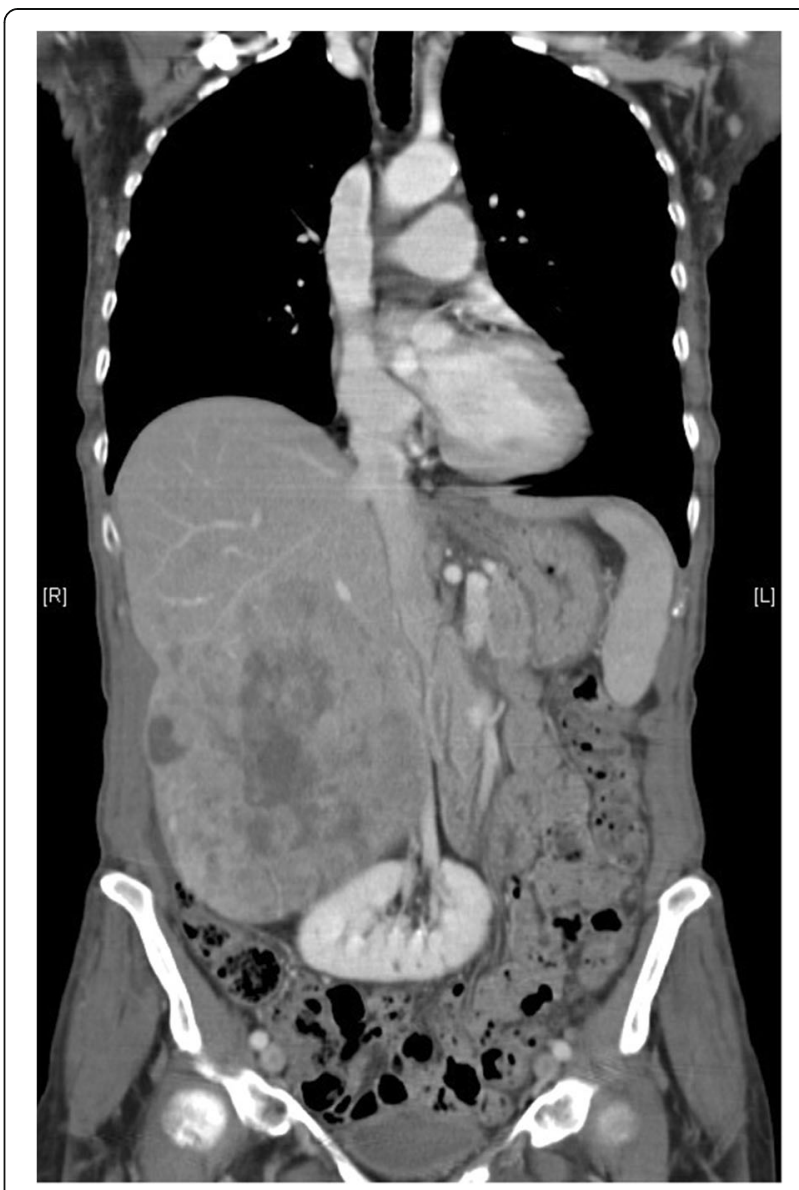

Figure 1 Computed tomography at initiation of pembrolizumab. Computed tomography scan of the liver before treatment with pembrolizumab was initiated after progression with the treatment of ipilimumab.

we performed a myocardial biopsy, which eventually confirmed the diagnosis of a lymphocytic myocarditis. An infiltration predominantly CD8 positive $\mathrm{T}$ cells was noted (Figure 3). Only a few FOXP3 positive, regulatory T cells were found showing signs of apoptosis (Figure 3). Serological and in situ analyses for cardiotropic viruses were negative. Thus, the diagnosis of immune-mediated myocarditis as irAE under pembrolizumab treatment was rendered.

We started a therapy with an AT2-receptor blocker (candesartan), a beta-blocker (bisoprolol), aldosteroneantagonist (spironolactone) and diuretics (torasemid) according to ACC/AHA guidelines as well as prednisone $2 \mathrm{mg}$ per kilogram body weight. Within two weeks, our patient showed a significant symptomatic recovery (NYHA II). This was in accordance with a normalization of the ECG (Figure 2) and echocardiographic improvement of left ventricular function (mildly reduced LVEF, 


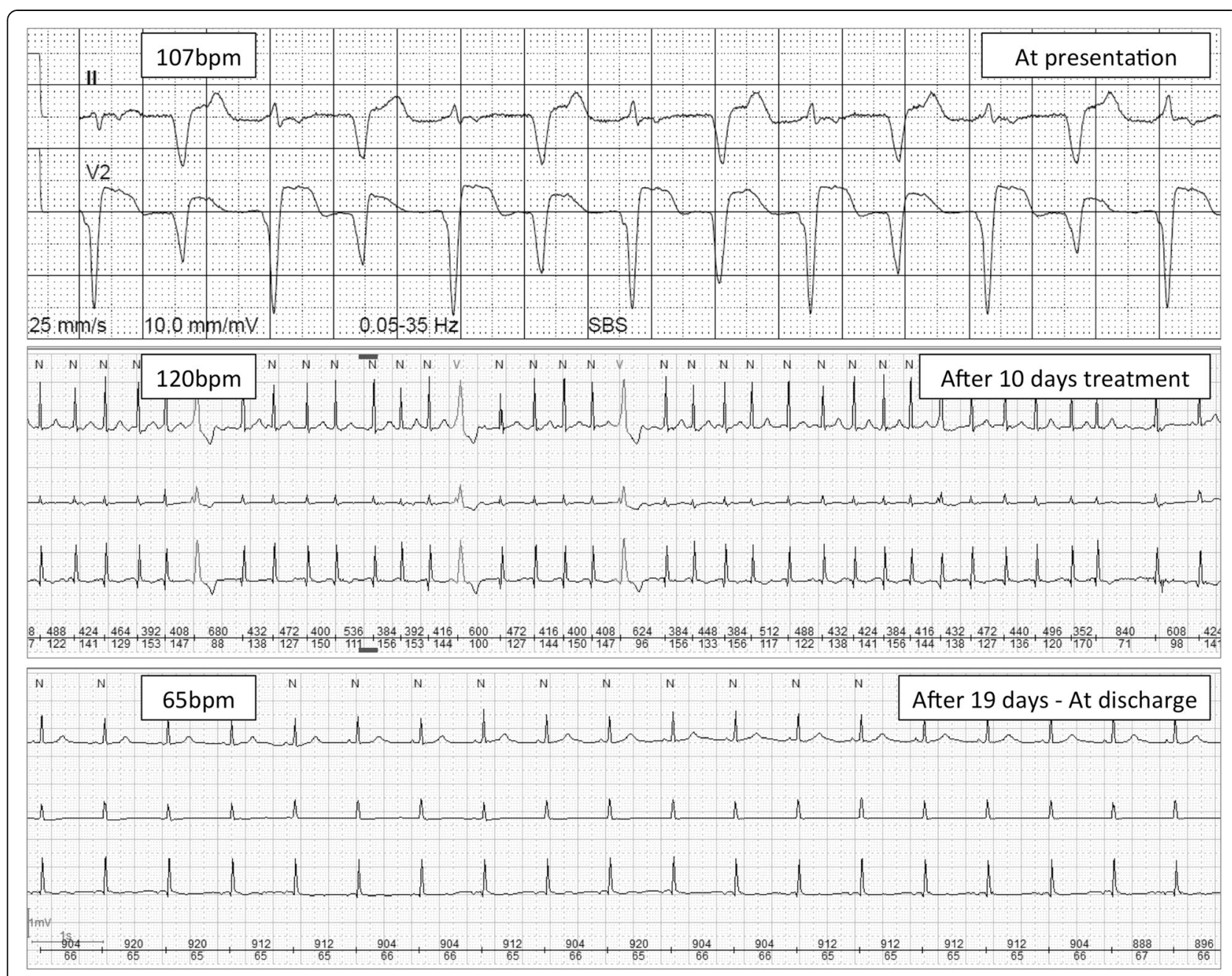

Figure 2 Electrocardiograms - At presentation and during follow-up. The electrocardiograms at admission, after 10 and 19 days of treatment are presented.

52\%). Also BNP and hs-TnT levels had significantly decreased (154 ng/L and $0.075 \mu \mathrm{g} / \mathrm{L}$, respectively). The patient could be discharged and anti-PD1 therapy was halted. No other specific anti-tumor therapy was initiated at the time.

\section{Discussion}

On September 4, 2014, the FDA granted accelerated approval of pembrolizumab (KEYTRUDA ${ }^{\circ}$ ) for the treatment of patients with unresectable or metastatic melanoma and disease progression following ipilimumab after the analysis of an expansion cohort of the KEYNOTE-001 trial of 173 patients [6]. The $2 \mathrm{mg}$ and $10 \mathrm{mg}$ per kilogram body weight regimens every 3 weeks showed similar response rates. Drug-related adverse events of any grade occurred in $82 \%$ and only $12 \%$ had grade 3 or 4 adverse events [6]. Grade 3 or 4 side effects due to immune stimulation or irAEs were only noted in 3 patients and included autoimmune hepatitis, maculopapular rash and pancreatitis [6]. In earlier studies of pembrolizumab, $4 \%$ of patients had pneumonitis, $1 \%$ grade 3 aminotransferase elevation, $8 \%$ hypothyroidism, $2 \%$ potentially autoimmune-mediated renal failure and one patient had autoimmune adrenalitis and hyperthyroidism [8]. Diarrhea developed in $20 \%$ of patients, but was usually mild and could be controlled without glucocorticoids [8]. Toxicities were less frequent in patients receiving $2 \mathrm{mg}$ per kilogram body weight every 3 weeks compared to $10 \mathrm{mg}$ every two or three weeks [8]. The toxicity profile and frequency of irAEs under pembrolizumab alone compared favorable to anti-CTLA-4 treatment with ipilimumab alone or in combination with anti-PD-1 therapy with nivolumab [11,12]. Similarly, pneumonitis, a potentially life-threatening complication, was noted less frequently 


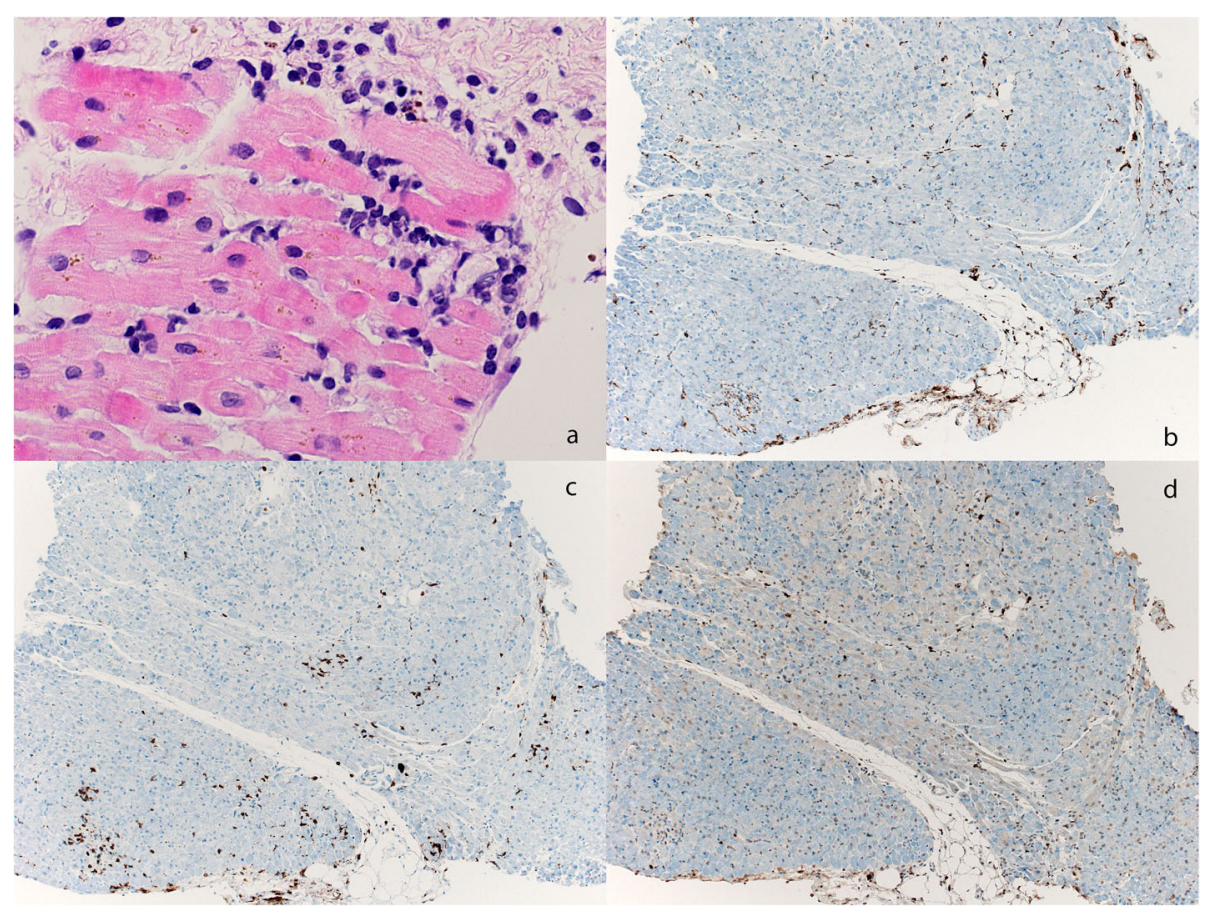

Figure 3 Histological analysis of endomyocardial biopsy. (a) Hematoxylin and eosin staining of the myocardial biopsy with focal mononuclear infiltrates. (b) Immunohistochemical analysis of CD68 macrophages. (c) Staining for CD8 positive T cells (d) and FOXP3 positive cells within the myocardium of the patient.

with pembrolizumab than in studies using nivolumab alone [10], although the tumor type likely plays a role in the toxicity profile. Future studies in other tumor types and combinations of immunostimulatory therapies that include pembrolizumab will certainly require careful monitoring and dose escalation schemes to avoid severe irAEs. Temporary or definitive discontinuation of immunostimulatory treatment and temporary immunosuppression can be an effective treatment in most cases and safety management guidelines are implemented in clinical trials and should also be used for patients treated outside trials [7].

There is no prior report on perimyocarditis or endocarditis after treatment with pembrolizumab $[6,8]$. While a similar spectrum of toxicities was observed with antiPD-1 antibody nivolumab $[5,7]$ and anti-PD-L1 antibody [9,13-15], only one case of myocarditis was reported in a phase one trial testing anti-PD-L1 antibody [9]. Myocarditis of non-infectious cause was previously described with anti-CTLA-4 treatment [3]. Histological analysis of autoimmune lesions and tumors after treatment with immune checkpoint inhibitors usually shows infiltration of effector CD8 T cells and reduction of regulatory FOXP3 positive $\mathrm{T}$ cells similarly as seen in the analysis of the myocardial biopsy in our patient [16] (Figure 3). Interestingly, various tissue-specific autoimmune conditions are observed in mice deficient for PD1 ( $\left.P d c d 1^{-1-}\right)$ [17]. These mice, particularly with a Balb/c background, die of heart disease, which is similar to human dilated cardiomyopathy. There is almost no inflammation in the heart of these mice and subsequent analyses revealed that auto-antibodies against cardiac troponin I are responsible for the disease [18]. In mice that are genetically predisposed to systemic autoimmunity, PD1 deficiency results in fatal myocarditis by 10 weeks of age that is reminiscent of $\mathrm{Ctla}^{-1-}$ mice [19]. Massive infiltration of both CD4 positive and CD8 positive $T$ cells and myeloid cells was found in hearts of those mice concomitant with the production of high-titer auto-antibodies against cardiac myosin. Subsequent experimental work clearly confirmed the important role for PD-1 in protecting the heart from $\mathrm{T}$ cell-mediated damage [20]. PD-1-deficient T lymphocytes caused enhanced disease with increased cytotoxic activity and inflammatory infiltrate.

Differential diagnosis of lymphocytic myocarditis and dilated cardiomyopathy includes infections with cardiotropic viruses. Among viral causes, enteroviruses and adenoviruses are historically common causes, but more recently, parvovirus $\mathrm{B} 19$, the recent $\mathrm{H} 1 \mathrm{~N} 1$ influenza pandemic, and human herpes virus 6 have become more prominent [21]. Although it carries some inevitable limitations, the endomyocardial biopsy remains the gold 
standard for the diagnosis of myocarditis [22]. According to the Dallas criteria, myocarditis is defined by lymphocytic infiltrates with or without myocyte necrosis. Lately, these criteria were challenged due to their limitations, including low sensitivity and high interobserver variability in interpretation of biopsy samples $[22,23]$. Therefore, advances in immunohistochemistry and PCR analyses of the endomyocardial specimens improved diagnostic accuracy for myocarditis [22,23]. In the presented case, we performed extensive diagnostics and excluded a viral cause for myocarditis. It is therefore very likely that the myocarditis of the patient was a result of immune stimulation due to PD-1 blockade with pembrolizumab.

\section{Conclusion}

We report here an autoimmune myocarditis as a side effect of an anti-PD-1-antibody, completely resolving after a therapy with high-dose corticosteroids. To our knowledge, it is the first time an autoimmune myocarditis under pembrolizumab treatment is reported. It is a documented side effect of other checkpoint-inhibitors, as for example ipilimumab and in one case with anti-PDL1 antibody, but not in anti-PD-1-antibodies like pembrolizumab or nivolumab. Our report should raise awareness for de novo cardial dysfunction in patients under PD-1 blockade. Approval of pembrolizumab and nivolumab by the FDA for the treatment of melanoma will lead to the use of these antibodies in a broader patient population with more concomitant diseases. Further ongoing studies and experience with patients outside of trials will provide more information about such rare side effects.

\section{Consent}

Written informed consent was obtained from the patient for publication of this case report and any accompanying images. A copy of the written consent is available for review by the Editor-in-Chief of this journal.

\section{Additional files}

Additional file 1: Transthoracal echocardiography at the time point of diagnosis of acute heart failure due myocarditis. Movie clip of apical 4 chamber (A4C) view.

Additional file 2: Transthoracal echocardiography at the time point of diagnosis of acute heart failure due myocarditis. Movie clip of parasternal long axis (PLAX) view.

Additional file 3: Transthoracal echocardiography at the time point of diagnosis of acute heart failure due myocarditis. Movie clip of parasternal short axis (PSAX) view.

\section{Competing interests}

H.L. received travel grants from Bristol-Myers Squibb. A.Z. received research funding from Roche Glycart consultant fees and travel grants from Roche, Bristol-Myers Squibb (BMS) and Merck, Sharp and Dohme (MSD).

\section{Authors' contributions}

$\mathrm{HL}, \mathrm{CB}$ and $\mathrm{AZ}$ treated the patient and wrote the case report. OP and MB performed and analyzed the cardial imaging and helped with rendering the diagnosis. KG analyzed the myocardial biopsy and studied immune infiltrates by immunostaining. All authors read and approved the final manuscript.

\section{Acknowledgements}

We thank the patient and her family for letting us present her case in this report. We also thank Clemens Winterhalder for helping to treat the patient during her hospital stay on the Internal Medicine ward at the University Hospital in Basel.

\section{Author details}

'Division of Medical Oncology, University Hospital Basel, Basel, Switzerland. 2Division of Cardiology, University Hospital Basel, Basel, Switzerland. ${ }^{3}$ Institute of Pathology, University Hospital Basel, Basel, Switzerland. ${ }^{4}$ Department of Biomedicine, Cancer Immunology Laboratory, University of Basel, Basel, Switzerland.

Received: 11 February 2015 Accepted: 11 March 2015

Published online: 21 April 2015

\section{References}

1. Rosenberg SA. Progress in human tumour immunology and immunotherapy. Nature. 2001;411:380-4.

2. Page DB, Postow MA, Callahan MK, Allison JP, Wolchok JD. Immune modulation in cancer with antibodies. Annu Rev Med. 2014;65:185-202.

3. Hodi FS, O'Day SJ, McDermott DF, Weber RW, Sosman JA, Haanen JB, et al. Improved survival with ipilimumab in patients with metastatic melanoma. N Engl J Med. 2010;363:711-23

4. Robert C, Thomas L, Bondarenko I, O'Day S, Weber J, Garbe C, et al. Ipilimumab plus dacarbazine for previously untreated metastatic melanoma. N Engl J Med. 2011;364:2517-26.

5. Ansell SM, Lesokhin AM, Borrello I, Halwani A, Scott EC, Gutierrez M, et al. PD-1 Blockade with Nivolumab in Relapsed or Refractory Hodgkin's Lymphoma. N Engl J Med. 2015;372:311-9.

6. Robert C, Ribas A, Wolchok JD, Hodi FS, Hamid O, Kefford R, et al. Anti-programmed-death-receptor-1 treatment with pembrolizumab in ipilimumab-refractory advanced melanoma: a randomised dose-comparison cohort of a phase 1 trial. Lancet. 2014;384:1109-17.

7. Robert C, Long GV, Brady B, Dutriaux C, Maio M, Mortier L, et al. Nivolumab in Previously Untreated Melanoma without BRAF Mutation. N Engl J Med: Waxman IM; 2014

8. Hamid O, Robert C, Daud A, Hodi FS, Hwu WJ, Kefford R, et al. Safety and tumor responses with lambrolizumab (anti-PD-1) in melanoma. N Engl J Med. 2013;369:134-44.

9. Brahmer JR, Tykodi SS, Chow LQ, Hwu WJ, Topalian SL, Hwu P, et al. Safety and activity of anti-PD-L1 antibody in patients with advanced cancer. N Engl J Med. 2012;366:2455-65.

10. Topalian SL, Hodi FS, Brahmer JR, Gettinger SN, Smith DC, McDermott DF, et al. Safety, activity, and immune correlates of anti-PD-1 antibody in cancer. N Engl J Med. 2012;366:2443-54.

11. Wolchok JD, Kluger H, Callahan MK, Postow MA, Rizvi NA, Lesokhin AM, et al. Nivolumab plus ipilimumab in advanced melanoma. N Engl J Med. 2013;369:122-33

12. Kong YC, Flynn JC. Opportunistic autoimmune disorders potentiated by immune-checkpoint inhibitors Anti-CTLA-4 and Anti-PD-1. Front Immunol. 2014:5:206.

13. Herbst RS, Soria JC, Kowanetz M, Fine GD, Hamid O, Gordon MS, et al. Predictive correlates of response to the anti-PD-L1 antibody MPDL3280A in cancer patients. Nature. 2014:515:563-7.

14. Powles T, Eder JP, Fine GD, Braiteh FS, Loriot Y, Cruz C, et al. MPDL3280A (anti-PD-L1) treatment leads to clinical activity in metastatic bladder cancer. Nature. 2014;515:558-62.

15. Wolchok JD, Chan TA. Cancer: Antitumour immunity gets a boost. Nature. 2014;515:496-8.

16. Hodi FS, Mihm MC, Soiffer RJ, Haluska FG, Butler M, Seiden MV, et al. Biologic activity of cytotoxic T lymphocyte-associated antigen 4 antibody blockade in previously vaccinated metastatic melanoma and ovarian carcinoma patients. Proc Natl Acad Sci U S A. 2003;100:4712-7. 
17. Nishimura H, Okazaki T, Tanaka Y, Nakatani K, Hara M, Matsumori A, et al. Autoimmune dilated cardiomyopathy in PD-1 receptor-deficient mice. Science. 2001;291:319-22.

18. Okazaki T, Tanaka Y, Nishio R, Mitsuiye T, Mizoguchi A, Wang J, et al. Autoantibodies against cardiac troponin I are responsible for dilated cardiomyopathy in PD-1-deficient mice. Nat Med. 2003;9:1477-83

19. Wang J, Okazaki IM, Yoshida T, Chikuma S, Kato Y, Nakaki F, et al. PD-1 deficiency results in the development of fatal myocarditis in MRL mice. Int Immunol. 2010;22:443-52

20. Tarrio ML, Grabie N, Bu DX, Sharpe AH, Lichtman AH. PD-1 protects against inflammation and myocyte damage in T cell-mediated myocarditis. J Immunol. 2012:188:4876-84.

21. Sagar S, Liu PP, Cooper LTJ. Myocarditis. Lancet. 2012;379:738-47.

22. Kindermann I, Barth C, Mahfoud F, Ukena C, Lenski M, Yilmaz A, et al. Update on myocarditis. J Am Coll Cardiol. 2012;59:779-92.

23. Dennert R, Crijns HJ, Heymans S. Acute viral myocarditis. Eur Heart J. 2008;29:2073-82

\section{Submit your next manuscript to BioMed Central and take full advantage of:}

- Convenient online submission

- Thorough peer review

- No space constraints or color figure charges

- Immediate publication on acceptance

- Inclusion in PubMed, CAS, Scopus and Google Scholar

- Research which is freely available for redistribution 